\title{
Cure of Atypical Teratoid/Rhabdoid Tumor of the Central Nervous System: A Case Report
}

\author{
Chengming Xu, Congyan Wu, Meiqing Lou, Yaodong Zhao* \\ Department of Neurosurgery, Shanghai General Hospital of Nanjing Medical University, Shanghai, China \\ Email: *703873213@qq.com
}

How to cite this paper: $\mathrm{Xu}, \mathrm{C} . \mathrm{M} ., \mathrm{Wu}$, C.Y., Lou, M.Q. and Zhao, Y.D. (2020) Cure of Atypical Teratoid/Rhabdoid Tumor of the Central Nervous System: A Case Report. Case Reports in Clinical Medicine, 9, 15-21.

https://doi.org/10.4236/crcm.2020.91003

Received: December 10, 2019

Accepted: December 28, 2019

Published: December 31, 2019

Copyright $\odot 2020$ by author(s) and Scientific Research Publishing Inc. This work is licensed under the Creative Commons Attribution International License (CC BY 4.0).

http://creativecommons.org/licenses/by/4.0/

\begin{abstract}
Atypical teratoid/rhabdoid tumor (AT/RT) is an embryonic central nervous system tumor. It has a low incidence with a high degree of malignancy and a poor prognosis. Five years ago, we successfully treated a child with AT/RT. Treatment comprised total tumor resection, $6 \mathrm{MV}$ X 3D conformal radiotherapy (DT: $36 \mathrm{~Gy} / 18 \mathrm{FX}$ ) and six courses of chemotherapy, including teniposide $25 \mathrm{mg}(\mathrm{qd} \times 5 \mathrm{~d})$, ACNU $25 \mathrm{mg}(\mathrm{qd} \times 1 \mathrm{~d})$, vincristine $1 \mathrm{mg}(\mathrm{qd} \times 1 \mathrm{~d})$. There was no tumor recurrence after 5 years of follow-up. We adjusted the previous AT/RT regimen to make it more suitable for the individual treatment of this patient, and now the patient has achieved a cure. So we think this regimen is effective and it is worthy of recommendation.
\end{abstract}

\section{Keywords}

Atypical Teratoid/Rhabdoid Tumor, Case Report

\section{Introduction}

Atypical teratoid/rhabdoid tumor (AT/RT) is a rare embryonic tumor in the central nervous system. The tumor contains a mixture of rhabdoid cells, primitive neuroectoderm, epithelial tissues, and mesenchymal tissues. The incidence of AT/RT is low, but there is a high degree of malignancy and a poor prognosis. Cause of death is primarily tumor recurrence and metastasis. Imaging features of AT/RT such as bleeding, necrosis, and cystic changes can often be observed by MRI or CT. The most common cause of AT/RT is an abnormality in chromosome 22 with the deletions of 22q11.2. For the treatment of AT/RT, most scholars still advocate the combination of radiotherapy and chemotherapy after surgical resection. Five years ago, we treated one case of AT/RT. The patient underwent total tumor resection, conformal radiotherapy and chemotherapy. As there was no recurrence after 5 years of follow-up with no recurrence, we consi- 
dered that she had been cured. We obtained the consent of the patient's parents to report the case. The case is reported herein for sharing with peers.

\section{Case Report}

The patient, a 3-year-2-month-old girl, was admitted due to "headache with weakness of the double lower limbs for 7 days". The physical examination revealed the following: being conscious, normal myodynamia in the double upper limbs (i.e., grade V), and grade IV myodynamia in the double lower limbs. CT scan showed a space-occupying lesion at the midline of the brain with a density similar to brain tissue, along with hydrocephalus (Figure 1). MRI revealed large space-occupying lesions in the ventricle with clear borders. Subsequent to contrast agent administration, most of the mass was enhanced, but we also observed some irregular sheet-like non-enhancing areas (Figure 2). We performed a total tumor resection under general anesthesia. During the operation, we discovered a large tumor (approximately $5 \times 4 \times 1.8 \mathrm{~cm}$ ) characterized by soft tumor tissue and dark red color, implying rich blood supply and we removed all the tumor tissue we could see in the bilateral ventricle and the third ventricle. Postoperative pathological diagnosis determined that it was an atypical teratoid/rhabdoid tumor (WHO Grade IV) in the lateral ventricle. Immunohistochemistry examination showed the following expressions: CK (+/-), EMA (+/-), vimentin (+), Syn $(-/+)$, GFAP (+/-), Desmin(-), Olig2(-), NeuN(-), and INI-1(-). One monthafter operation, the patient received $6 \mathrm{MV} \times 3 \mathrm{D}$ conformal radiotherapy. However, after radiotherapy $(1.8 \mathrm{~Gy} \times 2 \mathrm{FX})$, the patient suffered from hydrocephalus and subdural effusion. Thus, a ventriculoperitoneal (VP) shunting was performed. Two months after VP shunting, the patient underwent $6 \mathrm{MV} \times 3 \mathrm{D}$ conformal radiotherapy again (DT: $36 \mathrm{~Gy} / 18 \mathrm{FX}$ ). At the conclusion of radiotherapy, chemotherapy was administered as follows: teniposide $25 \mathrm{mg}(\mathrm{qd} \times 5 \mathrm{~d})$,

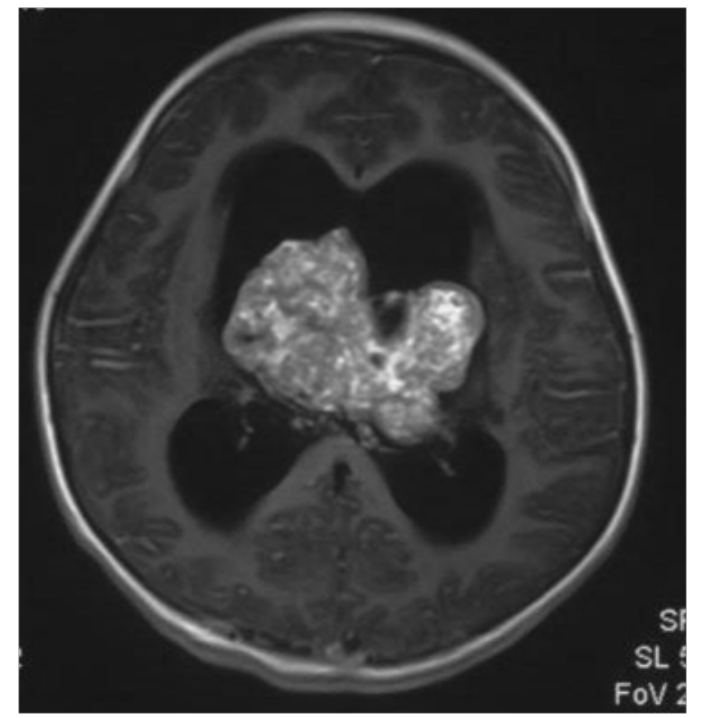

Figure 1. The preoperative CT scan shows a space occupying lesion at the midline of the brain, with adensity similar to brain tissue, along with hydrocephalus. 


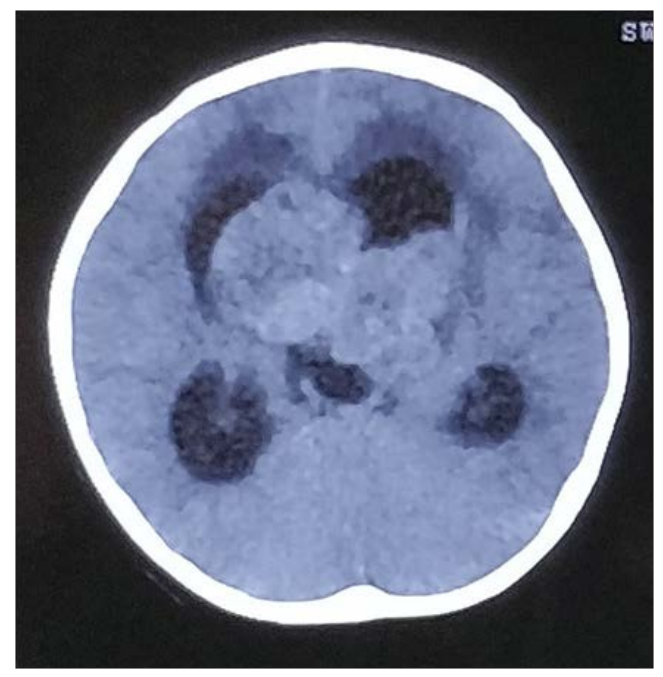

Figure 2. The MRI revealed large space-occupying lesions in the ventricle with clear borders. Most of the mass is enhanced subsequent to contrast agent administration. Some irregular sheet-like non-enhancing areas can also be observed.

ACNU $25 \mathrm{mg}(\mathrm{qd} \times 1 \mathrm{~d})$, and vincristine $1 \mathrm{mg}(\mathrm{qd} \times 1 \mathrm{~d})$ for a total of 6 courses. During chemotherapy, the patient did not have any adverse reactions to chemotherapy. In addition, the patients' general condition and cognitive activities were similar to those of children of the same age after chemotherapy. The patient was discharged home without other treatment and was advised to take valproic acid for antiepileptic action, and the patient was required to have regular reviews. At present, after 5 years of follow-up, the general condition of the patient is normal, and there is no developmental difference compared with healthy children of the same age. At the same time, we tested her neurocognition, like normal people, without being affected. MRI re-examination confirmed that there was no sign of tumor recurrence (Figure 3).

\section{Discussion}

$\mathrm{AT} / \mathrm{RT}$ in the central nervous system is a rare, highly malignant embryonic tumor named by Rorke et al. in 1996. The tumor contains a mixture of rhabdoid cells, primitive neuroectoderm, epithelial tissues, and mesenchymal tissues [1]. In 2007, WHO classified AT/RT, embryonic neuroectodermal tumor (PNET) and medulloblastoma as Grade IV embryonic tumor. The incidence of AT/RT is approximately 1.38 per 1 million, accounting for approximately $15 \%$ of CNS tumors in children under the age of 3 [2]. This tumor has a high degree of malignancy, poor prognosis, and high mortality. Cause of death is primarily tumor recurrence and metastasis [3]. According to statistics, AT/RT occurs most often in infants or children under 3 years old, and it is rare in adults. The incidence of $\mathrm{AT} / \mathrm{RT}$ is higher in males than in females, and there may be a hereditary component.

The clinical symptoms, signs, and imaging manifestations of AT/RT are varied, mainly due to factors such as the patient's age, the tumor location, and the 


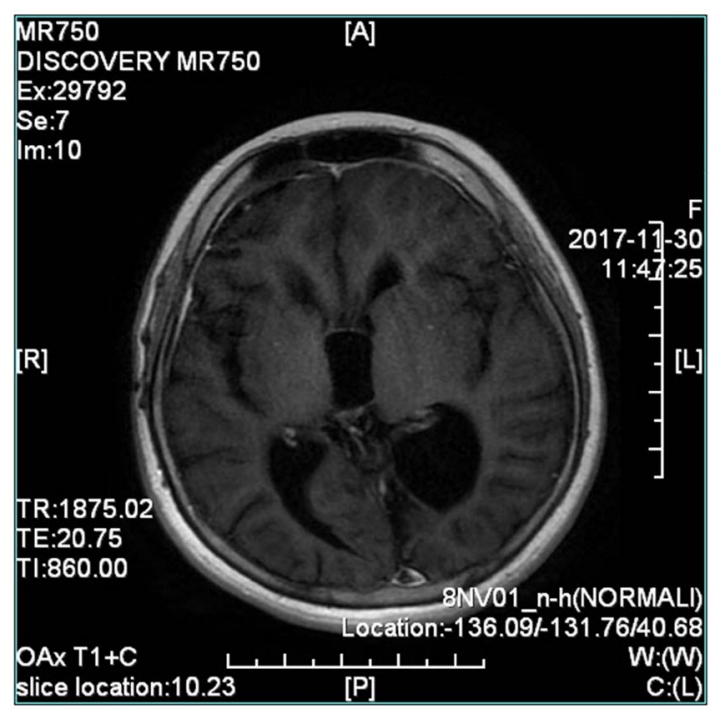

Figure 3. The MRI reexamination after five years shows no sign of tumor recurrence.

tumor size. Therefore, it often appears that different AT/RTs do not have obvious commonalities. The components of the tumor are complex. Despite similar imaging features, AT/RT needs to be differentiated from PNET and medulloblastoma. Characteristics of AT/RT include imaging features such as hemorrhage, necrosis, and cystic changes as observed through MRI or CT imaging, which are of great significance for differential diagnosis. There are also abundant vascular flow voids inside the tumor. This information can provide a certain value for differential diagnosis. Preoperative examination of the patient in this case showed significant space-occupying lesions at the midline of the brain by CT. During operation, the abundant blood supply of tumor tissues also conforms to the characteristics of AT/RT.

AT/RT has unique pathological morphology, including hemorrhage and necrosis. The physical boundary between the tumor and the surrounding brain tissue is unclear. Under light microscopy, the components of the tumor are revealed to be complex and diverse, with multiple differentiations and pleomorphisms. The morphology of the cells that make up the tumor tissue is diverse. The primitive round tumor cells are densely distributed in patches, while the epithelioid tumor cells are aggregated like nests. The primitive mesenchymal cells are distinct and have markedly deformed nuclei. The tumor is also characterized by a large number of nuclear fission phases, occasional nuclear delocalization, abundant cytoplasm, and scattered eosinophilic striated muscle (Figure 4). The most common histological indicators for AT/RT are cells positive for EMA, vimentin, NSE and GFAP [4]. In the case described herein, the tumor was vimentin (+) (Figure 5), EMA (+/-), Syn $(-/+)$, GFAP $(+/-)$, CK (+/-), and INI-1(-). These results are in accordance with previous studies on AT/RT.

The most common cause of AT/RT is an abnormality in chromosome 22 with the deletions of 22q11.2, where the gene INI-1 (also known as hSNF5 or SMARCB1) is located [5]. Currently, it is believed that abnormal expression of 


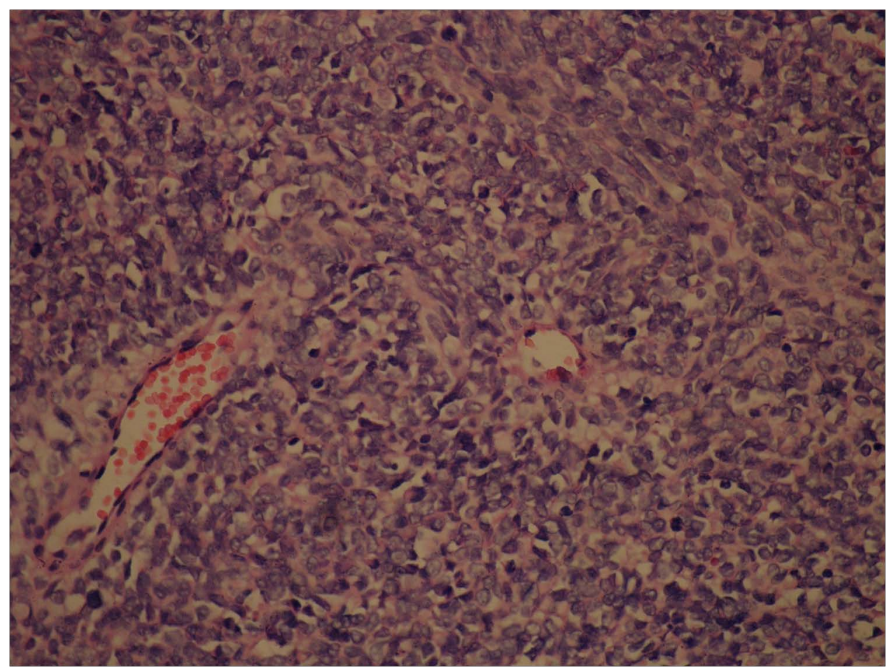

Figure 4. Hematoxylin and eosin staining of a tumor section at 400X magnification. Primitive round tumor cells are densely distributed in patchesand epithelioid tumor cells are aggregated like nests. The primitive mesenchymal components between some regions are distinct, and the nucleus of tumor cell is obviously deformed. We also observed a large number of nuclear fission phases, occasional nuclear delocalization, abundant cytoplasm, and scattered eosinophilic striated muscle.

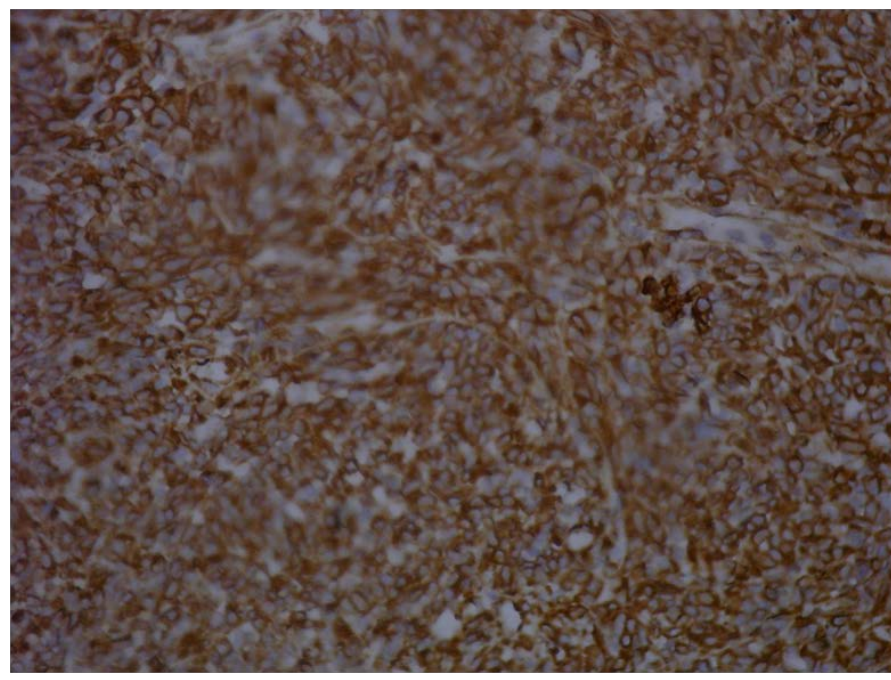

Figure 5. Vimentin is one of the most common histological indicators of AT/RT. This figure shows vimentin 400 times larger under an optical microscope.

INI-1plays an important role in the pathogenesis of AT/RT [6]. In this case, expression of INI-1 in the tumor tissue was absent, giving further evidence that INI-1 plays an important role in the pathogenesis of AT/RT.

For the treatment of AT/RT, most scholars still advocate surgical resection followed by a combination of radiotherapy and chemotherapy. Although studies have shown that AT/RT is sensitive to chemotherapy, there are currently no clear treatment guidelines. The clinical trials conducted by research centers use a treatment protocol of cyclophosphamide, vincristine and cisplatin after surgery [7]. While the prognosis of AR/RT has improved, the overall survival time (OS) 
remains approximately 11 to 24 months. In this case, we describe a modified chemotherapy plan of $25 \mathrm{mg}(\mathrm{qd} \times 5 \mathrm{~d})$ of teniposide, $25 \mathrm{mg}(\mathrm{qd} \times 1 \mathrm{~d})$ of ACNU and $1 \mathrm{mg}(\mathrm{qd} \times 1 \mathrm{~d})$ of vincristine, for a total 6 courses of chemotherapy. After five years of follow-up, the patient was found to be in good condition with no tumor recurrence. As the follow-up time was longer than the onset age of the patient plus 9 months, the disease was considered to be cured. In previous AT/RT cases, some patients were also cured, but most patients with this disease are still prone to relapse and metastasis, and the prognosis is poor [8]. In this case, the patient's pathology was clear and provided us with a basis for improvement of the regimens, so we changed the chemotherapy regimen and dose after consulting the literature and achieved good results.

Based on our experience and knowledge of the patient's disease, we adjusted the previous AT/RT treatment regimen to make it more suitable for the individual treatment of the patient, and has obtained certain curative effect. The patient is now cured. Therefore, we believe that this program is effective and worthy of recommendation. We hope that this program can serve as the basis and reference for the treatment of this disease.

\section{Not Published}

No contents of the paper may have been presented (not published) previously.

\section{Conflicts of Interest}

The authors declare no conflicts of interest regarding the publication of this paper.

\section{References}

[1] Radner, H., Blumcke, I., Reifenberger, G. and Wiestler, O.D. (2002) The New WHO Classification of Tumors of the Nervous System 2000. Pathologe, 23, 260-283. https://doi.org/10.1007/s00292-002-0530-8

[2] Austin, E.J. and Alvord Jr., E.C. (1988) Recurrences of Cerebellar Astrocytomas: A Violation of Collins' Law. Journal of Neurosurgery, 68, 41-47. https://doi.org/10.3171/jns.1988.68.1.0041

[3] Biggs, P.J., Garen, P.D., Powers, J.M. and Garvin, A.J. (1987) Malignant Rhabdoid Tumor of the Central Nervous System. Human Pathology, 18, 332-337. https://doi.org/10.1016/S0046-8177(87)80161-2

[4] Kitade, Y., Nakata, Y., Hirota, K., Maki, Y., Pabuccuoglu, A. and Torrence, P.F. (1991) 8-Methyladenosine-Substituted Analogues of 2-5A: Synthesis and Their Biological Activities. Nucleic Acids Research, 19, 4103-4108. https://doi.org/10.1093/nar/19.15.4103

[5] Bruch, L.A., Hill, D.A., Cai, D.X., Levy, B.K., Dehner, L.P. and Perry, A. (2001) A Role for Fluorescence in Situ Hybridization Detection of Chromosome 22q Dosage in Distinguishing Atypical Teratoid/Rhabdoid Tumors from Medulloblastoma/Central Primitive Neuroectodermal Tumors. Human Pathology, 32, 156-162. https://doi.org/10.1053/hupa.2001.21572

[6] Bruggers, C.S., Bleyl, S.B., Pysher, T., Barnette, P., Afify, Z., Walker, M. and Biegel, 
J.A. (2011) Clinicopathologic Comparison of Familial versus Sporadic Atypical Teratoid/Rhabdoid Tumors (AT/RT) of the Central Nervous System. Pediatric Blood \& Cancer, 56, 1026-1031. https://doi.org/10.1002/pbc.22757

[7] Tekautz, T.M., Fuller, C.E., Blaney, S., Fouladi, M., Broniscer, A., Merchant, T.E., Krasin, M., Dalton, J., Hale, G., Kun, L.E., et al. (2005) Atypical Teratoid/Rhabdoid Tumors (ATRT): Improved Survival in Children 3 Years of Age and Older with Radiation Therapy and High-Dose Alkylator-Based Chemotherapy. Journal of Clinical Oncology, 23, 1491-1499. https://doi.org/10.1200/JCO.2005.05.187

[8] Hilden, J.M., Meerbaum, S., Burger, P., Finlay, J., Janss, A., Scheithauer, B.W., Walter, A.W., Rorke, L.B. and Biegel, J.A. (2004) Central Nervous System Atypical Teratoid/Rhabdoid Tumor: Results of Therapy in Children Enrolled in a Registry. Journal of Clinical Oncology, 22, 2877-2884.

https://doi.org/10.1200/JCO.2004.07.073 